\title{
ESTUDIO ETNOBOTÁNICO DE LAS PLANTAS MEDICINALES EMPLEADAS POR LA COMUNIDAD RURAL DE ZAQUE-MUNICIPIO DE GACHETÁ, CUNDINAMARCA
}

\author{
Bibiana González - Marcela Mora \\ Licenciadas en Biología. Universidad Pedagógica Nacional. \\ Myriam Clavijo \\ Bióloga, MSc en Ciencias. Profesora Departameto de Biología. U.P.N.
}

\begin{abstract}
The etnobotánica applied as integral science in the handling of the vegetation and the culture, constituted an useful tool for the rescue and recognition of the popular knowledge and the traditions that still conserve the different sectors of the rural community of the sidewalk Drunkard. The contributions of this science transcend of the simple striped taxonómicos of plants "useful" toward the conception that the peasant possesses on the vegetable environment and the cultural baggage in which is unwrapped, with the purpose of waking up in the new generations the interest to recognize their cultural roots and to strengthen his identity like essential part iside the handling of the natural environment and of his conservation.
\end{abstract}

\section{RESUMEN}

La etnobotánica aplicada como ciencia integral en el manejo de la vegetación y la cultura, constituyó una herramienta útil para el rescate y reconocimiento de los saberes populares y las tradiciones que aún conservan los diferentes sectores de la comunidad rural de la vereda Zaque. Los aportes de esta ciencia trascienden de los simples listados taxonómicos de plantas "útiles" hacia la concepción que posee el campesino sobre el entorno vegetal y el bagaje cultural en el que se desenvuelve, con el fin de despertar en las nuevas generaciones el interés por reconocer sus raíces culturales y fortalecer su identidad como parte esencial dentro del manejo del entorno natural y de su conservación.

PALABRAS CLAVE

Etnobotánica - Plantas medicinales - Etnografía

\section{INTRODUCCIÓN}

El municipio de Gachetá se encuentra ubicado al nororiente del Departamento de Cundinamarca, a una altura de 1.745 m.s.n.m.; cuenta con cerca de 14.000 habitantes, de los cuales un 70\% corresponden a la zona rural (GUZMÁN, 1993). La investigación se llevó a cabo contando con la participación de la comunidad rural de la vereda de Zaque. El objetivo principal estuvo enfocado hacia la recopilación de la información etnobotánica, principalmente del recurso floral de uso medicinal en la zona. De esta forma, empleando algunas herramientas de la Etnografía se logró un buen acercamiento a la visión que tiene el campesino de su entorno natural y se recopilé una valiosa información acerca del uso que ha venido dando la comunidad a la flora de su región en materia medicinal. De igual forma, logró 
evidenciarse la necesidad de inculcar en las nuevas generaciones la conciencia de una identidad cultural que permita mantener vivos los conocimientos y costumbres acumulados por dicha comunidad a través de los años.

\section{ANTECEDENTES}

La etnobotánica en Colombia es una ciencia relativamente nueva. Su desarrollo se inicia a partir de 1860 con la obra "Botánica Indígena" del Doctor Florentino Vezga; a partir de entonces, se han llevado a cabo otras investigaciones principalmente con las comunidades indígenas del país con el fin de realizar inventarios de flora útil con predominio de plantas medicinales, alimenticias, rituales y para vivienda. Posteriormente se han realizado investigaciones en otros sectores de la población colombiana que incluyen comunidades campesinas, plazas de mercado de grandes ciudades, entre otros, con referencias culturales acerca del uso de los recursos vegetales.

En este sentido, vale la pena destacar las obras "Plantas útiles de Colombia' (1956) de Enrique Pérez Arbeláez y "Flora Medicinal de Colombia" (1 .974) de Hernando García Barriga (ZULUAGA, 1994).

\section{JUSTIFICACION}

Haciendo una revisión sobre los estudios florísticos de la zona, se encontró que son muy escasos los trabajos existentes sobre este tema y que ha sido muy poca la importancia que se ha dado al conocimiento de la riqueza biológica de esta región. Partiendo de este punto y dado el reciente auge de la medicina tradicional y el actual interés de la Organización Mundial de la Salud por la recuperación de los conocimientos populares sobre plantas medicinales como alternativa para la Atención Primaria en Salud principalmente en los países más pobres, además de la preocupación de organizaciones como la Unión Internacional para la Conservación (IUCN) y el Fondo Mundial para la Vida Silvestre (WWF) por la conservación de los recursos naturales del planeta, se hace necesario desarrollar estrategias de investigación que permitan rescatar y conservar los conocimientos populares que acerca de este tema poseen nuestros pueblos (ZULUAGA, 1994). En este contexto, la Etnobotánica como ciencia del estudio de las relaciones entre el hombre y las plantas, constituye un valioso aporte al descubrimiento de las relaciones que se entretejen a diario entre el hombre y la naturaleza, generando conocimientos que contribuyen al fortalecimiento de una conciencia de protección y conservación de nuestra riqueza biológica y cultural.

\section{OBJETIVOS O PROPOSITO}

El objetivo principal del estudio consistió en recopilar, organizar y divulgar la información sobre el recurso vegetal de uso medicinal en una comunidad rural de la vereda de Zaque, en el municipio de Gachetá (Cund.). De esta forma, el trabajo contribuyó al conocimiento de la flora regional, partiendo de los saberes populares de los mismos habitantes de la zona, obteniendo información a través de informantes clave, abuelos, padres de familia y estudiantes de dos de las escuelas de la vereda. El trabajo incluye la determinación taxonómica de los especimenes vegetales colectados, junto con una revisión bibliográfica sobre los estudios fitoquímicos existentes de los mismos. 


\section{METODOLOGIA}

Teniendo en cuenta que el objetivo y la realización de este trabajo implica directamente un acercamiento a un grupo social determinado, cuyas características propias y únicas, exige una revisión minuciosa de la metodología a emplearse, se tuvieron en cuenta algunas de las técnicas de investigación social más empleadas en este tipo de trabajos. De esta forma, la Etnografía que tiene como fin último el estudio sociocultural o el estilo de vida de una sociedad, describiendo las creencias y prácticas del grupo, mostrando cómo las diversas partes de la comunidad contribuyen a crear la cultura como un todo unificado y consistente (BRIONES, 1988), constituyó una base importante para el desarrollo de nuestro trabajo. "La Etnografía se interesa por lo que la gente hace, cómo se comporta, cómo interactúa. Se propone describir sus creencias, valores, perspectivas, motivaciones, y el modo en que todo ello se desarrolla o cambia dentro del grupo y desde las perspectivas de sus miembros. Lo que cuentan son sus significados e interpretaciones” (MARTíNEZ,, 1997).

El trabajo se realizó con una muestra de 63 personas de la comunidad, dentro de las cuales 5 corresponden a abuelos, 18 a padres de familia, 36 a estudiantes de 40 y 50 grado de Educación Básica y 4 informantes clave. Para la recolección de información se emplearon Entrevistas semiestructuradas realizadas a los abuelos, padres de familia e informantes clave y talleres aplicados a los estudiantes. Como técnicas de registro de información se emplearon audiograbaciones, videograbaciones, notas de campo, fichas etnobotánicas, además de una observación participativa constante.

De acuerdo con la secuencia de la investigación pueden definirse 5 fases, así:

1. Fase inductiva: Revisión bibliográfica, reconocimiento de la zona, indagación y conexión con personas clave dentro de la comunidad.

2. Fase de recolección de la información: Entrevistas semiestructuradas, Talleres, colección del material vegetal de uso medicinal, elaboración de fichas etnobotánicas y realización de videograbaciones para la posterior edición del video.

3. Fase de categorización: Revisión, organización y categorización de la información obtenida.

4. Fase interpretativa: Análisis de la información estableciendo las relaciones etnobotánicas implícitas en ésta. Interpretación y análisis de la información concerniente al uso de las plantas como recurso para el cuidado de la salud.

5. Fase de divulgación: Diseño y estructuración de una cartilla ilustrativa de la flora medicinal de la zona. Retroalimentación a la comunidad.

\section{RESULTADOS}

A través de este trabajo se logró vislumbrar en buena medida la importancia de las relaciones que se establecen entre el campesino y su ambiente, específicamente con su entorno vegetal, a través del cual obtiene su alimento, ingresos económicos, calidad de agua, entre muchos otros beneficios. Sin embargo, más allá del simple utilitarismo, el campesino otorga un profundo significado a la naturaleza desde el 
punto de vista estético, cultural y emocional; el campesino sabe que gracias a los recursos que le brinda su entorno obtiene una calidad de vida que no podría, por su condición, encontrar en las grandes ciudades; es por ello que se considera como parte inseparable de su ambiente, siendo consiente que de su manejo y sus acciones depende que éste pueda brindar a sus descendientes los mismos beneficios.

En cuanto al conocimiento sobre el uso de plantas medicinales, se encontró que éste tipo de saberes son manejados principalmente por personas que en su mayoría sobrepasan los 55 años; éstas personas fueron consideradas como informantes clave, y a pesar de que fue un grupo pequeño de sólo 4 personas, sus aportes resultaron bastante significativos. A este grupo corresponden personas que han tenido siempre una relación directa con la naturaleza, manifestada en su interés por contribuir en el mantenimiento de la salud de su comunidad desde distintos ámbitos.

A partir de la información recopilada por medio de los informantes clave, padres de familia, abuelos y niños de la comunidad, se encontraron 71 especies de plantas de uso medicinal en la zona, de las cuales se colectaron e identificaron 57 de ellas, distribuidas en 36 familias botánicas. En el Cuadro $\mathrm{N}^{0} 1$ puede verse una parte de la información etnobotánica aportada por los informantes clave, como ilustración de la forma en que se recopiló y organizó la información proveniente de cada uno de los subgrupos con que se realizó el trabajo dentro de la vereda.

\begin{tabular}{|c|c|c|c|}
\hline \multicolumn{4}{|c|}{ En la vereda de Zaque } \\
\hline $\begin{array}{l}\text { NOMBRE } \\
\text { DELAPLANTA }\end{array}$ & uso & PARTE UTILIZADA & $\begin{array}{l}\text { FORMA DE } \\
\text { PREPARACIÓN }\end{array}$ \\
\hline SAUCO & $\begin{array}{l}\text { ENFERMEDADES } \\
\text { RESPIRATORIAS }\end{array}$ & HOJAS Y FLORES & INFUSION \\
\hline SAUCE & HEMORRAGIAS & HOJAS & CATAPLASMA \\
\hline MOQUILO & INFLAMACIÓN & HOSAS & ZUMO \\
\hline VERBENA & FIEBRE & HOJAS, TALLO, FLORES & DECOCCIÓN \\
\hline CHIPACA AMARILLA & DOLOR DE ESTÓMAGO & RAIZ & DECOCCIÓN \\
\hline POMPAS & INFLAMACIÓN & HOJAS, FLORES, FRUTO & BAÑOS \\
\hline VALERIANA & DOLOR DE MUELA & TODALAPLANTA & CATAPLASMA \\
\hline CORDONCILLO & INFLAMACIÓN & HOJAS & BAÑOS \\
\hline HAYUELO & INFLAMACIONES & HOJAS, TALLO & LECHO \\
\hline CEDRO NOGAL & DESINFECTANTE & HOSAS & BAÑO \\
\hline TILO & BRONQUITIS & FLOR & INFUSIÓN \\
\hline BREVO & EMBARAZO, PARTO & HOSAS, FRUTO & DECOCCIÓN Y BAÑOS \\
\hline CAFETERO & MATRIZ & HOJAS & DECOCCION \\
\hline RUDA & $\begin{array}{l}\text { PROVOCALA } \\
\text { MENSTRUACIÓN }\end{array}$ & HOJAS & INFUSIÓN \\
\hline
\end{tabular}

Partiendo de los conocimientos sobre la flora medicinal recopilados a través de la comunidad, se categorizaron las plantas de acuerdo a la frecuencia de su uso; dentro de las plantas de uso frecuente encontramos aquellas reconocidas por informantes clave, padres de familia y por los niños quienes dan la pauta para co- 
rroborar que son plantas que realmente se mantienen aún en su tradición (Ver cuadro $\mathrm{N}^{0}$ 2). . En el caso de las plantas de uso semi-frecuente podemos encontrar las especies mencionadas principalmente por los padres de familia y los informantes clave (Ver cuadro N"3). Finalmente dentro de la categoría de plantas de uso poco frecuente, incluimos aquellas especies que conocen exclusivamente los informantes clave, quienes manejan una información mucho más amplia sobre las plantas medicinales (Ver cuadro $\mathrm{N}^{0} 4$ ).

Cuadro $\mathrm{N}^{\circ}$ 2. Plantas de uso frecuente

\begin{tabular}{|l|l|}
\hline \multicolumn{1}{|c|}{ NOMBRE VULGAR } & \multicolumn{1}{c|}{ NOMBRE CIENTÍFICO } \\
\hline CALÉNDULA & Calendula officinalis L. \\
\hline MANZANILLA MATRICARIA & Anthemis nobilis L. \\
\hline TORONJIL & Melissa officinalis L. \\
\hline YERBABUENA & Mentha sp. \\
\hline ALBAHACA & Ocimum michrantum \\
\hline ALTAMISA & Ambrosia cumanensis HBK. \\
\hline RUDA & Ruta graveolens L. \\
\hline ZÁBILA & Aloe vera L. \\
\hline PAPAYA & Carica papaya L. \\
\hline SAUCO & Sambucus nigra L. \\
\hline APIO & Apium graveolens L. \\
\hline CIDRÓN & Aloysia triphylla (L' Her) Britton \\
\hline VERBENA & Verbena litoralis HBK. \\
\hline POLEO & Satureia brownii (Sw.) Briquetier \\
\hline
\end{tabular}

Cuadro $\mathrm{N}^{\circ} 3$. Plantas de uso semifrecuente

\begin{tabular}{|l|l|}
\hline \multicolumn{1}{|c|}{ NOMBRE VULGAR } & \multicolumn{1}{c|}{ NOMBRE CIENTÍFICO } \\
\hline SAUCE & Salix humboldtiana Willd. \\
\hline GUABA & Phytolacca bogotensis HBK \\
\hline MASTRANTO & Salvia paliifolia HBK. \\
\hline LLANTÉN & Plantago major L. \\
\hline DIENTEDELEÓN & Taraxacum officinalis \\
\hline PEPAMORA OYERBAMORA & Solanum americanum Mill. \\
\hline UCHUVA & Physalis peruviana L. \\
\hline BREVO & Ficus carica L. \\
\hline TOTE & Rhynchospora nervosa \\
\hline LIMONARIA & Cymbopogon citratus \\
\hline
\end{tabular}


Cuadro $\mathbf{N}^{\circ}$ 2. Plantas de uso poco frecuente

\begin{tabular}{|c|c|}
\hline NOMBRE VULGAR & NOMBRE CIENTÍFICO \\
\hline COLA DE CABALLO & Equisetum bogotense HBK \\
\hline CICUTA & Conium maculatum L. \\
\hline POMPAS & Asclepias fruticosa L. \\
\hline PALITARIA & Pilea parietaria (L.) Blume \\
\hline PAICO & Chenopodium ambrosioides L. \\
\hline LAUREL & Laurus nobilis L. \\
\hline CURAPOO AGUACATE & Persea americana Mill. \\
\hline CORDONCILLO & Piper aduncum L. \\
\hline CANELÓN & Peperomia ilaloensis Sod. \\
\hline MOSTAZA & Brassica rapa \\
\hline YEMA DE HUEVO CHUPA HUEVO & Sedum praeatlum D.C. \\
\hline ARRAYÁN & $\begin{array}{l}\text { Myrcianthes leucoxyla (Ortega) } \\
\text { Mc Vaugh. Fieldiana. }\end{array}$ \\
\hline HINONO & Foeniculum vulgare L. \\
\hline ESTRANCADERA & Hypoestes sp. \\
\hline CHIPACABLANCA & Bidens pilosa L. \\
\hline CHIPACA AMARILLA & Bidens andicola \\
\hline MANGO & Mangifera indica L. \\
\hline MIRTO & Solanum pseudocapsicum L. \\
\hline PEPAMORA O YERBAMORA & Solanum americanum Mill. \\
\hline ZARZAPARRILLA & Smilax sp. \\
\hline ALCAPARRO & Senna sp. \\
\hline SÁNDALO & Pelargonium graveolens L' Her. \\
\hline QUENOROOO & Hibiscus rosa-sinensis L. \\
\hline MORADITA & Cuphea racemosa Spreng. \\
\hline LENGUA DE VACA O ROMAZA & Rumex crispus L. \\
\hline GAQUE & Clusia alata Planch. \& Triana. \\
\hline SALVAVIDAS & Borreria ocymoides (Burm.) \\
\hline
\end{tabular}

De acuerdo con la revisión bibliográfica sobre la fotoquímica de las especies colectadas e identificadas, encontramos que la gran mayoría de las plantas de uso medicinal que emplea la comunidad rural de Zaque, han venido siendo utilizadas desde tiempo atrás y cuentan con un amplio estudio sobre su efecto medicinal y sus principios activos; es el caso de las plantas aromáticas introducidas principalmente del viejo continente como la manzanilla, cidrón, mejorana, toronjil y otras plantas medicinales introducidas como la ruda, la zábila, el laurel y el eucalipto. Estas plantas son ampliamente reconocidas, cultivadas y de uso frecuente entre los habitantes de la zona quienes encuentran en ellas un recurso importante para el tratamiento de enfermedades y dolencias comunes. De acuerdo con la 
revisión bibliográfica de estas especies, se encontró una correspondencia entre el uso medicinal dado por la comunidad de Zaque y el uso reportado por los autores consultados.

En algunos otros casos, el uso medicinal dado por la comunidad de Zaque a algunas plantas no se encontró reportado en la bibliografía, lo cual podría significar otros usos potenciales para éstas plantas que aún no han sido estudiados, o también usos folklóricos que corresponden sólo a creencias o costumbres de los habitantes de la zona. Esto para el caso de plantas como el Llantén (Plantago major), la papaya (Carica papaya), el mastranto (Salvia palaefolia), el diente de león (taraxacum officinale), el mango (Mangifera indica), la cicuta (Conium maculatum), la zarzaparrilla (Smilax regelli), el sauce (Salix humboldtiana), el curapo (Persea americana), la 'mostaza (Brassica rapa), la moradita (Cuphea racemosa), el arrayán (Myrcianthes leucoxyla), y la pepa-mora o yerbamora (Solanum americanum).

Para algunas de las plantas reconocidas principalmente por los informantes clave y catalogadas como de uso poco frecuente entre los habitantes de esta zona, no se encontraron usos, ni estudios fitoquímicos acerca de sus principios activos en la bibliografía consultada, por lo tanto, éstas podrían constituir un punto interesante para nuevos estudios enfocados hacia la potencialidad medicinal de éstas especies. Es el caso del Salvavidas (Borreria ocymoides), Pompas (Asclepia fruticosa), Yema de huevo (Sedum praeatlum) y Gaque (Clusia alata).

Otras plantas como el Cenelón (Peperomia ilaloensis) han sido poco estudiadas en nuestro país, aunque se sospecha favorablemente de sus propiedades medicinales (CORPAS, 1992).

En el aspecto pedagógico del trabajo, los talleres realizados con los niños mostraron el amplio conocimiento que ellos manejan acerca de la vegetación, conociendo los nombres y algunas propiedades de ciertas plantas que han aprendido a través de las enseñanzas de sus padres. Sin embargo, los niños manejan información acerca de las plantas medicinales empleadas con mayor frecuencia en sus hogares, desconociendo los nombres y propiedades de muchas otras plantas de su región. También es importante resaltar la influencia e importancia que ha tenido la vegetación en la vida del campesino, expresada en los cuentos, adivinanzas, coplas y refranes realizados por el grupo de niños que participó en la investigación, en donde puede verse la riqueza cultural que conservan aún estas comunidades Campesinas.

\section{CONCLUSIONES}

El trabajo en Etnobotánica constituye una valiosa herramienta para acercarnos con mayor profundidad a las ciencias biológicas desde la mirada de quienes conviviendo a diario con la naturaleza tienen mucho que enseñarnos y aportarnos en nuestra formación como docentes y como personas. A través de este trabajo puede verse la inmensa riqueza de los saberes populares que permanecen aún en nuestros pueblos y que merecen una pronta atención que frene la pérdida acelerada de éstos conocimientos generada por los cambios sociales y culturales de la vida moderna.

En cuanto al conocimiento sobre la flora de uso medicinal en la zona, se recopiló una valiosa información que incluye un buen número de plantas y formas de uso; los resultados de éste trabajo pueden constituir una buena base para posteriores 
investigaciones sobre la fitoquímica de éstas especies, principalmente de aquellas acerca de las cuales no hay aún ningún tipo de estudio.

Es importante resaltar que a través de los materiales logrados en el trabajo (vídeo - cartilla) puede lograrse una retroalimentación hacia la comunidad, de tal forma que ellos puedan hacerse conscientes de la riqueza biológica y cultural que poseen y puedan de esta forma valorarla y conservarla a través de las generaciones.

\section{BIBLIOGRAFÍA}

Briones, G. 1988. Métodos y técnicas avanzadas de investigación aplicadas a la educación y a las ciencias sociales. ICFES. Módulo 2. Bogotá.

Corpas, J. y otros. 1992. Plantas medicinales (Compendio de farmacología vegetal). Segunda Edición. Fondo Editorial de la Escuela de Medicina Juan N. Corpas. Santafé de Bogotá.

García, H. 1974. Flora medicinal de Colombia. Botánica médica. Segunda Edición. Tercer Mundo Editores. Tomo I. Santafé de Bogotá.

1974. Flora medicinal de Colombia. Botánica médica. Segunda Edición.

Tercer Mundo Editores. Tomo II. Santafé de Bogotá.

1974. Flora medicinal de Colombia. Botánica médica. Segunda Edición. Tercer Mundo Editores. Tomo III. Santafé de Bogotá.

Guzmán, M. 1993. Gachetá, 400 años. Editorial de Cundinamarca (EDICUNDI).

Martínez, M. 1997. La investigación cualitativa etnográfica en educación. Círculo de Lectura Alternativa Ltda. Bogotá, Colombia.

Sanabria, 0. 1991. La etnobotánica colombiana actual: implicaciones y tendencias. En: Memorias del tercer simposio colombiano de etnobotánica, INCIVA. Cali.

Yepes, S. 1951. Introducción a la etnobotánica colombiana. Sociedad Colombiana de Etnología Bogotá.

Zuluaga, G. 1994. El aprendizaje de las plantas en la senda de un camino olvidado. Seguros Bolívar. Santafé de Bogotá, D.C. 